depressed. Experimental study was made by the authors on the influence of plasmin upon steroid hormon or hypothermia which was applied therapeutically to the case of brain injury or brain edema. Experimental groups were divided as follows:

A) normothermic group

B) normothermic + brain injury group

C) normothermic + brain injury + administrate steroid horman group

D) hypothermic group

E) hypothermic + brain injury group

F) hypothermic + brain injury + administrate steroid horman group

Furthermore the followings were measured for this experiment.

1) plasmic activity, 2) euglobulin plasmin, 3) plasma antiplasmin, 4) whole plasmin, 5) on injuried brain tissue plasminogen

Predonisolon $5 \mathrm{mg} / \mathrm{kg} /$ day was administered. As the result, the author found that fibrinolytic activity varied mostly in the group B and was depressed in the group $F$.

From our experimental results, the authors thinks that administration of steroid hormon and application of hypothermia are the best therapy for the severe case which brain injury from the point of view to protect the brain barrier from disturbance due to brain injury.

\title{
24. Transtentorial Herniation in connection with Acute Head Injuries
}

\author{
Kenji Tanaka and Kiyoshi Sato \\ Neurosurgical Clinic, Department of Surgery, Juntendo University \\ School of Medicine \\ Michio INUI \\ Tokyo-To Medical Examiner Office
}

Since Adolph Meyer in 1920, the transtentorial herniation associated with an increased supratentorial pressure has been discussed by many investigators over the past 40 years. A few papers have been reported in Japan, because of pausity of the systematic investigation for the transtentorial herniation of acute brain injuries in the statistical and pathological studies.

Materials were collected from Tokyo-To Medical Examiner Office and Juntendo University Hospital for a period from 1964 to 1965. Ninety-six brains were examined by the necropsy in the cases of injuries. In 96 cases, 
there were 84 cases of head injuries and the rest of 12 cases did not show any cranial injuries. Of 84 cases, 69 presented the transtentorial herniation. This transtentorial herniations were classified in 4 types by the authors, i.e. Type-I is unilateral anterior herniation; Type-II is bilateral anterior herniation; Type-III is bilateral or unilateral herniation, involving anterior and posterior herniation; Type-IV is unilateral complete herniation. In this series, the Type-I was $16 / 69$; the Type-II was $28 / 69$; the Type-III was $8 / 69$; the Type-IV was $17 / 69$.

Ten cases of Type-I, brain damage without hematoma, were noticed to be located in the orbital surbace of frontal lobe and the tip of temporal lobe. Sixteen cases of Type-II, hematoma alone or hematoma with brain damage, were found to be located in the frontal base to its convex. Seven cases of Type-III, hematoma alone or hematoma with brain damage which diffusely were in the both hemispheres of the cerebrum from the base to its convex. Seventeen cases of Type-IV, epi- or subdural hematoma with or without brain damage, which were diffusely from the base to the convex of either hemisphere of cerebrum.

Twenty-six tonsillar herniations were found associating with transtentorial herniations. In this series, 5 were found in Type-I; 8 in Type-II; 3 in Type-III; 10 in Type-IV. Almost all of the tonsillar herniations were mild in degree. Sixteen cingular herniations were also noted in this series. Eleven of which were noted in the Type-IV. Only 3 cases of unilateral sphenoidal herniations were encountered in this study. All of them were found in Type-II.

Seven cases of infratentorial injuries were found in this study. All of them were associated with supratentorial injuries. No tonsillar or upward herniation were encounted without transtentorial herniation.

All types of transtentorial herniation were noted to be associated or combined with following lesions such as narrowed interpeduncular space, twisted or distorted cerebral peduncle, shifted brainstem, and dorsally displaced peduncle. In cases of large transtentorial herniation, following consequences were noted; compression of the aqueduct of Sylvius, pressure necrosis of cerebral peduncle by the tentorial notch. In Type-III and TypeIV, the cerebral peduncle was markedly flattened out due to compression effect of the large herniation and the antero-posterior diameter of cerebral peduncle was increased.

The pressure groove of incisura tentori in the anterior lobe of cerebellum were noticed in 10 cases of 69 transtentorial herniations. This groove may have resulted from an increasing supratentorial pressure. The authors hypothesized following explanations for the caudal displacement of brainstem, and emphasis was placed on a downward bulging of the tentorium cerebelli. 
This is a sign of displacement of the brainstem through the cerebellum by the caudal bulging of tentorium cerebelli due to markedly increased supratentorial pressure. When the tentorium extends caudally, the crumen and its adjascent tissue protrude and engage in the ambient cistern, simultaneously, the displaced cerebelli and herniated temporal lobe obliterate the basal cistern completely. In addition to this, if the aqueduct of Sylvius become compressed, aforementioned mechanisms play an acceleration of increment of supratentorial pressure.

In preparation for the microscopical studies of the temporal and occipital lobe which are supplied mainly by the posterior cerebral artery (P.C.A.), we studied the pathomorphological aspects of the compressions of this artery in connection with the herniated tissue, cerebral peduncle and free edge of the tentorium cerebelli. The results are as follows: The posterior communicating artery is depressed inferomedially in every type of the herniation in this series. Although it may be easily stretched in the basilar cistern but is never compressed to any direction. In Type-I, the P.C.A. is easily compressed in the anterior portion between anterior herniated tissue (mainly uncus) and the ipsilateral pyramis of cerebral peduncle. And anterior temporal branches of the P.C.A. are again compressed between the anterior herniated tissue and the anterior portion of the free edge of the tentorium cerebelli. In Type-II, same type of the compression of the arteries are occur bilaterally. In Type-III, the P.C.A. is compressed by the two portion, i.e. in the first place, between anterior herniated tissue and the pyramis of the peduncle and, in the second place, between posterolateral portion of the central peduncle and posterior herniated tissue, which compression is accelerated by bilateral herniations. Simultaneously, the branches of P.C.A., the anterior and posterior temporal branches and the calcarine branch are compressed between the herniated tissues and the free edge of the temtorium cerebelli. In Type-IV, the mechanism of the compression of the artery is slightly different from that of the another types. The posterior cerebral aetery is embraced in the superior and medial surface of the large herniated tissue involving the all portion of the hippocampal gyrus, so that compression of the posterior cerebral artery is milder. However, the every branches of the P.C.A. are strongly compressed between large herniated tissue and entire free edge of the tentorium cerebelli.

Microscopic findings of this studies will be reported following papers. 